\title{
Bitterness Reduction of Naringin and Limonin by $\beta$-Cyclodextrin
}

\author{
Akira Konno, Masaru MisaKi, Jun Toda, \\ Takeo WADA and Katsuharu YASUMATSU \\ Food Research Laboratories, Food Products Division, \\ Takeda Chemical Industries, Ltd., Osaka 532, Japan \\ Received September 28, 1981
}

\begin{abstract}
Taste interactions of $\beta$-cyclodextrin with naringin or limonin, which are bitter tasting constituents of citrus fruits and their products, were evaluated by psychophysical methods. The mechanism of interaction of $\beta$-cyclodextrin with naringin or limonin was explained by both the solubilities of the bitter tasting compounds and the changes brought about by proton shifts within the $\beta$-cyclodextrin.

In the presence of $0.5 \% \beta$-cyclodextrin, the bitterness of naringin or limonin was reduced to approximately half of what it was without $\beta$-cyclodextrin. This reduction in bitterness by $\beta$ cyclodextrin was also observed in the citrus juice itself.

The solubilities of naringin or limonin increased with the addition of $\beta$-cyclodextrin. In a series of ${ }^{1} \mathrm{H}$ NMR spectra of $\beta$-cyclodextrin in $\mathrm{D}_{2} \mathrm{O}$, the low-field triplet signals assigned to the $\mathrm{H}-3$ and H-5 protons, which are located within the cavity of $\beta$-cyclodextrin, showed a gradual shift to the higher-field values with increases in the molar ratio of naringin to $\beta$-cyclodextrin. It was concluded that this bitterness reduction was due to the formation of an inclusion complex between the $\beta$ cyclodextrin and the naringin or limonin.
\end{abstract}

The bitterness of citrus fruits and their products is caused primarily by either of two constituents-naringin and limonin. Naringin is said to be the bitter tasting constituent of grapefruit, natsudaidai, buntan oranges and so forth. ${ }^{1)}$ On the other hand, limonin is the bitter tasting component of navel oranges, valencia oranges and iyo oranges, among others. ${ }^{2,3)}$ These bitter compounds are often necessary components for the acceptable flavors of citrus juices. However, when the intensity of the bitter taste in the citrus juice is too strong to be acceptable, it is necessary to reduce this bitterness in commercial citrus products. Unitl now reduction or elimination of the bitterness has been accomplished either by enzymatic treatment, ${ }^{4,5)}$ or by the adsorption of the bitter constituents on polyamide and polyvinylpyrrolidone resin powders. ${ }^{6}$ )

Cyclodextrins, also known as Schardinger dextrins, are cyclic oligosaccharides composed of 6 or more glucose units with $\alpha-1,4$-linkages. They are capable of forming inclusion com- plexes with various organic and inorganic compounds. It is this characteristic property that has interested many researchers. ${ }^{7)}$

In previous papers, ${ }^{8,9)}$ it has been reported that the turbidity of canned mandarin orange syrup, which is caused by hesperidin, can be reduced by increasing the solubility of hesperidin through the formation of an inclusion complex with $\beta$-cyclodextrin. Preliminary experiments have also indicated that $\beta$-cyclodextrin can reduce the bitterness of both naringin, which is chemically analogous to hesperidin, and limonin. ${ }^{10,11)}$

The interactions of $\beta$-cyclodextrin with these bitterness components were evaluated by psychophysical methods, and the mechanism of their interactions with $\beta$-cyclodextrin was also studied using both solubility measurements of the bitter tasting compounds and changes brought about by proton shifts within the $\beta$ cyclodextrin. 


\section{MATERIALS AND EXPERIMENTAL METHODS}

Materials. Naringin was purchased from Tokyo Kasei Kogyo Co. Ltd. The limonin, extracted from the seeds of Citrus natsudaidai, was kindly supplied by Prof. Nakabayashi of Shizuoka University. The $\beta$-cyclodextrin used in these experiments was purchased from Nihon Shokuhin Kako Co. as CELDEX NT, moisture 11.0\%, purity $99.0 \%$.

\section{Experimental methods.}

A. Sensory evaluation.

1. Evaluation of the bitterness intensity of naringin or limonin solutions. Aqueous solutions 0.01, 0.02 and $0.04 \%$ naringin and naringin containing $0.5 \% \quad \beta$-cyclodextrin were prepared. The bitterness intensities of these solutions were evaluated according to Scheffe's paired comparison. ${ }^{12)}$ In addition, aqueous solutions of 0.75 and $1.50 \mathrm{mg} \%$ limonin and limonin containing $0.5 \% \beta$-cyclodextrin were prepared and evaluated as above.

2. Evaluation of the taste quality of citrus juices.

a. Natsudaidai juice. Concentrated natsudaidai juice was reconstituted, and then sucrose was added until the Brix degree $(\mathrm{Bx})$ reached $13^{\circ}$. This reconstituted juice contained $45.0 \mathrm{mg} \%$ naringin. The juice was divided into three portions: one, a control sample without $\beta$-cyclodextrin, and the other two containing $0.3 \%$ and $0.5 \% \beta$-cyclodextrin, respectively. The bitter, sweet and sour taste intensities of the samples containing $\beta$-cyclodextrin were organoleptically evaluated by comparison with the same qualities of the control, using a 7-point rating scale.

b. Iyo juice. The juice was prepared from the fruit of Citrus iyo, diluted twofold and sucrose was added until the Bx reached $13^{\circ}$. This juice was divided into two portions: one, untreated, and the other heated to $93^{\circ} \mathrm{C}$. Each of these portions was further subdivided into two parts: one without $\beta$-cyclodextrin, and one with $0.5 \% \beta$-cyclodextrin added. The bitter, sweet and sour taste intensities of these four samples were evaluated by Scheffe's paired comparison. The subjects participating in this sensory evaluation were staff members of the Food Research Laboratories, Takeda Chemical Industries Ltd. All personnel were accustomed to this type of evaluation.

$B$. Measurement of the solubility of naringin and limonin. In the case of naringin, aqueous suspensions of $3 \times 10^{-3} \mathrm{M}$ naringin containing different amounts of $\beta$-cyclodextrin were prepared. These suspensions were heated in a boiling water bath, and then kept at $5^{\circ} \mathrm{C}$ for 10 days. After this refrigeration period, the suspensions were filtered through Toyo Filter Paper No. 5B, and the concentration of naringin in each filtrate was determined using the method of Davis as modified by Nakabayashi et al. ${ }^{13)}$

The same procedure as described above was also followed for limonin; however, its original concentration in the suspension was $1 \times 10^{-4} \mathrm{M}$. The concentration of limonin was then determined by the $p$-dimethylamino- benzaldehyde assay as modified by Misawa et al. ${ }^{14)}$

C. Proton nuclear magnetic resonance $\left({ }^{1} H N M R\right)$. A fixed amount of naringin was placed in an NMR tube ( $5 \mathrm{~mm}$ of diameter), and a $\mathrm{D}_{2} \mathrm{O}$ solution containing $15.2 \mathrm{~mm} \beta$-cyclodextrin was added with mixing. The resulting solution was incubated in a $50 \sim 70^{\circ} \mathrm{C}$ water bath for $10 \mathrm{~min}$ and then used as the sample for NMR measurement. Sodium-2,2-dimethyl-2-silapentano-5-sulfonate (DSS) was the reference substance. The proton shifts in the ${ }^{1} \mathrm{H}$ NMR spectra of $\beta$-cyclodextrin were determined at $23^{\circ} \mathrm{C}$, using a JEOL-FX-200 spectrometer.

\section{RESULTS AND DISCUSSION}

\section{A. Reduction of sensory bitterness by $\beta$ - cyclodextrin}

1. Bitterness reduction of naringin or limonin in aqueous solutions. Figure 1 illustrates the relationship between the perception of bitterness and the actual concentration of naringin. Sensory bitterness is directly proportional to the logarithm of the concentration, thus following Fechner's law. Sensory scales developed using Scheffe's paired comparison are category scales, while those following Stevens' power law are magnitude scales. ${ }^{11)}$

In the presence of $0.5 \% \beta$-cyclodextrin, the bitterness intensity of naringin was reduced to approximately $50 \%$ of that without $\beta$-cyclodextrin. A similar results as shown in Fig. 2 was observed with limonin solutions containing $0.5 \% \quad \beta$-cyclodextrin. However, $\beta$ cyclodextrin had no significant effect on the

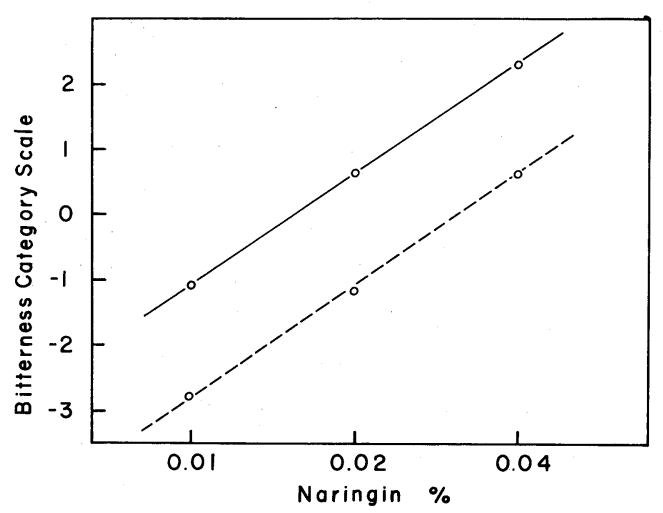

Fig. 1. The Effects of Added $\beta$-Cyclodextrin on Naringin Bitterness.

_- without $\beta$-cyclodextrin; -.---., with $0.5 \% \quad \beta$ cyclodextrin. 


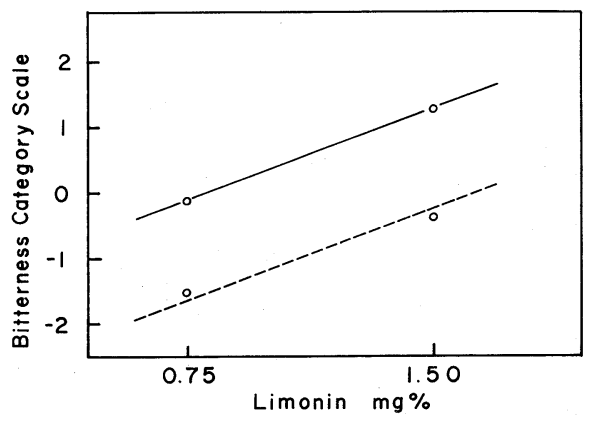

Fig. 2. The Effects of Added $\beta$-Cyclodextrin on Limonin Bitterness.

- , without $\beta$-cyclodextrin; --.---, with $0.5 \% \quad \beta$ cyclodextrin.

intensities of the bitter tastes of quinine hydrochloride, caffeine and phenylthiourea. ${ }^{11)}$

2. Bitterness reduction of juice products by $\beta$ cyclodextrin. As the bitterness of naringin or limonin in aqueous solution can be reduced by $\beta$-cyclodextrin, so it seemed possible to eliminate or reduce the bitterness of some citrus juices by the addition of $\beta$-cyclodextrin.

Naringin has been identified as the bitter tasting constituent in Citrus natsudaidai. ${ }^{1)}$ The bitter, sweet and sour taste intensities of Citrus natsudaidai juices containing $\beta$-cyclodextrin were organoleptically evaluated by comparison with those without $\beta$-cyclodextrin. As Table I indicates, the bitterness intensity was reduced significantly in the presence of 0.3 and $0.5 \%$ of $\beta$-cyclodextrin.

Citrus iyo, usually eaten fresh in Japan, contains limonin as the bitter component. ${ }^{2,3)}$ Again, the bitter, sweet and sour taste in- tensities of the juices of Citrus iyo with and without $\beta$-cyclodextrin were evaluated. The results obtained are given in Table II. Limonin is produced from its precursor during storage or heat treatment. In this experiment, however, the increase in bitterness due to heating was not remarkable. However, with the addition of $\beta$-cyclodextrin, bitterness was reduced significantly and an increase in sweetness was also observed.

\section{B. Effect of $\beta$-cyclodextrin on the solubilities of naringin and limonin}

The reduction of the bitterness of naringin and limonin in the presence of $\beta$-cyclodextrin was thought to be the result of the formation of an inclusion complex between $\beta$-cyclodextrin and the naringin or limonin. Solubility measurements are usually the simplest methods to demonstrate the formation of in-

TABle I. TASTE Modification OF Citrus natsudaidai JUICE BY $\beta$-CYClODEXTRIN

\begin{tabular}{lcc}
\hline & \multicolumn{2}{c}{$\beta$-Cyclodextrin, $\%$} \\
\cline { 2 - 3 } & 0.3 & 0.5 \\
\hline Bitterness $^{a}$ & $-0.50(*)^{b}$ & $-0.63(* *)^{b}$ \\
Sweetness $^{a}$ & 0.04 & 0.08 \\
Sourness $^{a}$ & -0.13 & 0.00
\end{tabular}

a. Scale values are the differences in intensity from that of the control, $n=24$; the larger the value, the more intense the taste.

$b \quad *$ and $* *$ denote statistical significance at 0.05 and 0.01 confidence levels, respectively.

Table II. TASte Modification of Citrus iyo Juice By $\beta$-CyClodeXtrin

\begin{tabular}{|c|c|c|c|c|c|}
\hline & \multicolumn{4}{|c|}{$\beta$-Cyclodextrin } & \multirow{3}{*}{ Remarks $^{b}$} \\
\hline & 0 & 0.5 & 0 & 0.5 & \\
\hline & \multicolumn{2}{|c|}{ Unheated } & \multicolumn{2}{|c|}{ Heated } & \\
\hline Bitterness $^{a}$ & 0.53 & -0.63 & 0.81 & -0.72 & $* *$ \\
\hline Sweetness ${ }^{a}$ & -0.34 & 0.41 & -0.47 & 0.41 & $* *$ \\
\hline Sourness $^{a}$ & 0.00 & -0.03 & 0.19 & -0.16 & \\
\hline
\end{tabular}

a The principal characteristics compared in Scheffe's analysis of variance, $2 r=8$. The larger the value, the more intense the taste.

$b \quad * *$ indicates statistical significance at the 0.01 level of confidence. 


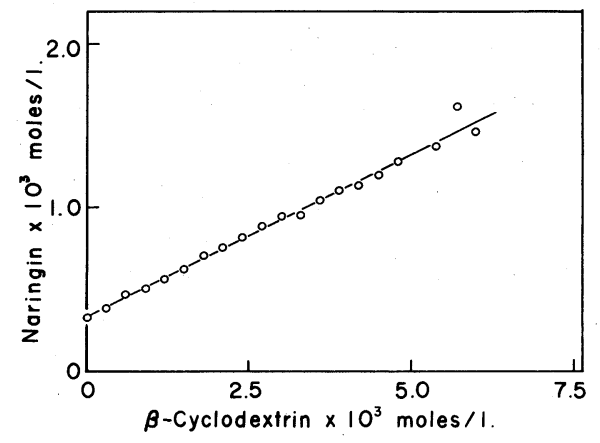

FIG. 3. Effect of $\beta$-Cyclodextrin on the Solubility of Naringin at $5^{\circ} \mathrm{C}$.

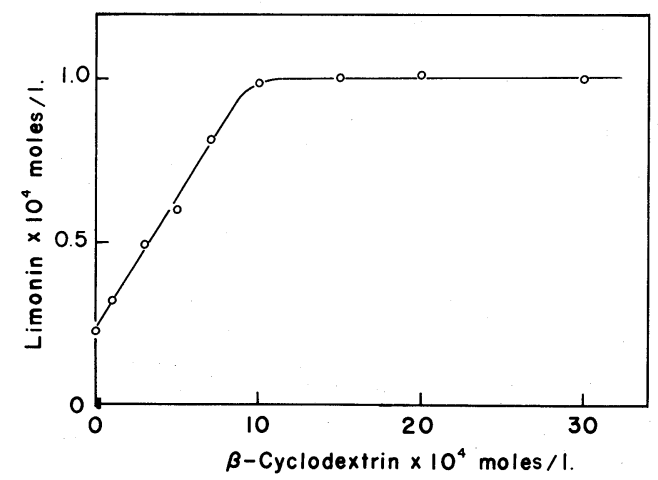

FIG. 4. Effect of $\beta$-Cyclodextrin on the Solubility of Limonin at $5^{\circ} \mathrm{C}$.

clusion complexes between cyclodextrin and various organic and inorganic compounds. ${ }^{15)}$

Figures 3 and 4 illustrate the relationship between the amount of dissolved naringin or limonin and added $\beta$-cyclodextrin. The concentrations of dissolved naringin and limonin increased with increasing $\beta$-cyclodextrin concentration. Thus, the increases in solubility of naringin or limonin with the addition of $\beta$ cyclodextrin can be attributed to the formation of inclusion complexes between the naringin or the limonin and $\beta$-cyclodextrin.

\section{NMR spectra demonstrating the formation of inclusion complexes}

Solubility measurements, however, do not provide direct evidence of the formation of inclusion complexes. On the other hand, NMR spectra have been recognized as direct methods of verification of the formation of in-

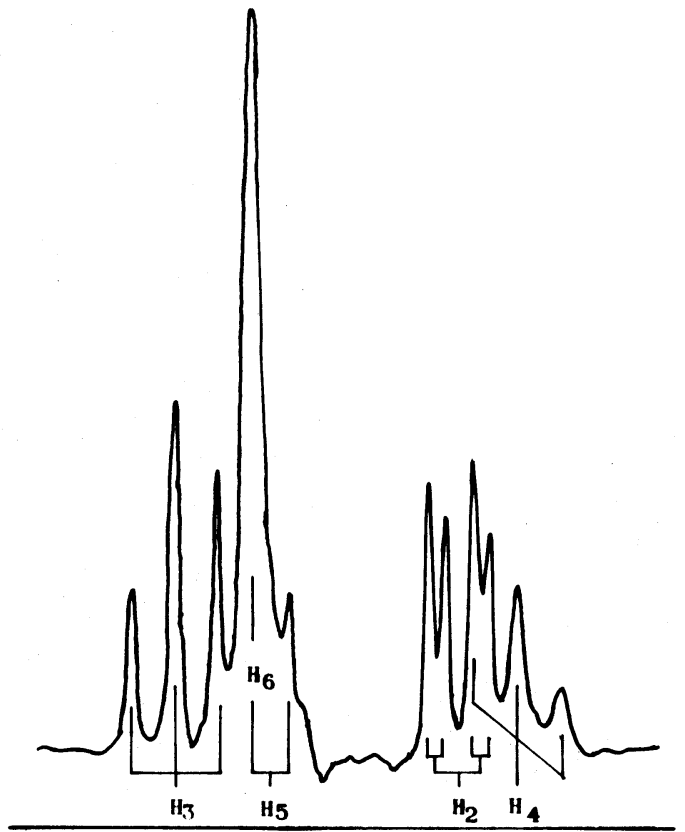

Fig. 5. The ${ }^{1} \mathrm{H}$ NMR Spectrum (200 MHz) of $15.2 \mathrm{~mm}$ $\beta$-Cyclodextrin in $\mathrm{D}_{2} \mathrm{O}\left(23^{\circ} \mathrm{C}, \mathrm{pD}=7\right)$.

Sweep width, $2 \mathrm{kHz}$.

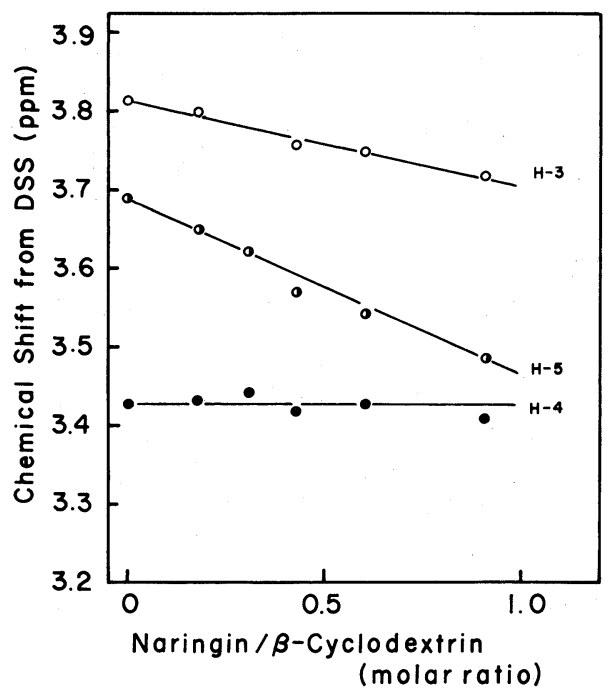

FIG. 6. Chemical Shifts of $\beta$-Cyclodextrin Protons in $\mathrm{D}_{2} \mathrm{O}$ at $23^{\circ} \mathrm{C}$, Relative to DSS as an External Reference, as Caused by the Presence of Different Concentrations of Naringin.

clusion complexes. ${ }^{16)}$ The cyclodextrin molecule is doughnut-shaped. It has been reported that if certain "guest" compounds mig- 
rate into the interior of the cavity of the cyclodextrin, the $\mathrm{H}-3$ and $\mathrm{H}-5$ protons, located within the cavity, will be strongly shielded. ${ }^{16,17)}$

Figure 5 shows the $200 \mathrm{MHz}{ }^{1} \mathrm{H}$ NMR spectrum of $\beta$-cyclodextrin in $\mathrm{D}_{2} \mathrm{O}$ at $23^{\circ} \mathrm{C}$, while Fig. 6 indicates the relationship between the chemical shifts of the $\mathrm{H}-3, \mathrm{H}-4$ and $\mathrm{H}-5$ protons, measured relative to DSS as the external reference, and the molar ratios of naringin relative to $\beta$-cyclodextrin. From examination of the ${ }^{1} \mathrm{H}$ NMR spectra of $\beta$ cyclodextrin in $\mathrm{D}_{2} \mathrm{O}(15.2 \mathrm{mM})$, it can be seen that the low-field triplets, assigned to the H-3 proton, shifted gradually to higher-field values with increasing molar ratios of naringin relactive to $\beta$-cyclodextrin. The $\mathrm{H}-5$ proton signals also shifted from their initial positions. The signals of the other protons $(\mathrm{H}-1, \mathrm{H}-2, \mathrm{H}-4$ and $\mathrm{H}-6$ ), which are located on the exterior or near the cavity of $\beta$-cyclodextrin molecule, were not affected by the addition of naringin. It was also observed that the spectrum generated by the methyl group of the rhamnose moiety of the naringin molecule was not affected by the presence of $\beta$-cyclodextrin.

These chemical shifts of the $\beta$-cyclodextrin protons definitively establish that the phenyl ring of naringin is positioned within the $\beta$ cyclodextrin cavity. From these results, it can be concluded that $\beta$-cyclodextrin forms this inclusion complex, not with the rhamnose moiety, but with phenyl ring of naringin. In the case of limonin, examination of ${ }^{1} \mathrm{H}$ NMR spectra was impossible because of the low solubility of limonin. But from solubility measurements, it is conceivable that $\beta$-cyclodextrin can form a similar complex with limonin.

Let us assume that the molar ratio of naringin or limonin to $\beta$-cyclodextrin is $1: 1$ in the complex, and that the solubility of naringin or limonin is independent of $\beta$-cyclodextrin concentration. Then let S, C and SC be defined as free naringin or limonin, free $\beta$-cyclodextrin and the complex formed between naringin or limonin and $\beta$-cyclodextrin in the solution, respectively. Their interaction is shown in the following equation:

$$
\mathrm{S}+\mathrm{C} \rightleftarrows \mathrm{SC}
$$

From the data given in Figs. 3 or 4 , the concentration of $\mathrm{S},[\mathrm{S}]$, can be determined from the solubility of naringin or limonin in solution in the absence of $\beta$-cyclodextrin. Since the total concentrations of naringin or limonin, $\mathrm{S}_{\mathrm{T}}$, and $\beta$-cyclodextrin, $\mathrm{C}_{\mathrm{T}}$, are given in advance, the concentrations of $\mathrm{SC},[\mathrm{SC}]$, and $\mathrm{C},[\mathrm{C}]$, can be calculated as follow:

$$
\begin{gathered}
{[S C]=\left[S_{T}\right]-[S]} \\
{[C]=\left[C_{T}\right]-[S C]=\left[C_{T}\right]-\left(\left[S_{T}\right]-[S]\right) .}
\end{gathered}
$$

As a result, the association (stability) constant between naringin or limonin and $\beta$-cyclodextrin can now be defined and calculated by the following equation:

$$
\mathrm{K}=[\mathrm{SC}] /[\mathrm{S}][\mathrm{C}] \text {. }
$$

The calculated values of $\mathrm{K}$ for naringin and limonin, obtained from the data in Figs. 3 and 4 , were $7.5 \times 10^{2} \mathrm{M}^{-1}$ and $3.9 \times 10^{3} \mathrm{M}^{-1}$, respectively. These values approximate those obtained from thermodynamic measurements for other molecules complexed with $\beta$-cyclodextrin $\left(K=2.5 \times 10^{3} \mathrm{M}^{-1}\right.$ for phenol, $1.2 \times$ $10^{2} \mathrm{M}^{-1}$ for benzoic acid, $1.0 \times 10^{3} \mathrm{M}^{-1}$ for 4 nitrophenol etc.). ${ }^{18)}$

It has been not reported before that $\beta$ cyclodextrin reduces the bitterness of naringin or limonin and forms inclusion complexes with naringin or limonin. However, in our experiments, it was found that this bitterness was reduced by the addition of $\beta$-cyclodextrin. Bitterness reduction by $\beta$-cyclodextrin was also shown in citrus juices in which naringin or limonin was said to be the bitter tasting constituent. It was then shown that the formation of inclusion complexes between $\beta$-cyclodextrin and naringin or limonin was responsible for the decrease in bitterness.

Acknowledgments. The authors wish to express their appreciation to Professor T. Nakabayashi of Shizuoka University who kindly supplied the limonin. They also acknowledge the technical assistance of Messrs. M. Miyawaki and K. Kawamura. 


\section{REFERENCES}

1) T. Nakabayashi, J. Agric. Chem. Soc., 35, 945 (1961).

2) R. H. Higby, J. Am. Chem. Soc., 60, 3013 (1938).

3) Y. Bessho, M. Kodama, Y. Matsumoto and S. Kubo, Presented at the Meeting of the Nishi-Nippon Branch of the Agric. Chem. Soc. Jpn., Matsuyama, Oct., 1980.

4) F. M. Roumbouts and W. Pilnik, Process Blochem., 3, 9 (1978).

5) S. V. Ting, J. Agric. Food Chem., 6, 547 (1958).

6) F. P. Griffiths, U. S. Patent 3,463,763 (1969).

7) D. French, Adv. Carbohydrate Chem., 12, 189 (1957).

8) A. Konno, M. Miyawaki, M. Misakı and K. Yasumatsu, Nippon Shokuhin Kogyo Gakkaishi, 29, 255 (1982).

9) M. Misaki, A. Konno, M. Miyawaki and K. Yasumatsu, Nippon Shokuhin Kogyo Gakkaishi, 29, 228 (1982).
10) A. Konno, M. Miyawaki, M. Misaki and K. Yasumatsu, Agric. Biol. Chem., 45, 2341 (1981).

11) J. Toda, M. Misaki, A. Konno, T. Wada and K. Yasumatsu, "The Quality of Foods and Beverages," Vol. 1, ed. by G. Charalambous and G. Inglett, Academic Press Inc., New York, 1981, p. 19.

12) H. Scheffe, J. Am. Statist. Assoc., 47, 381 (1952).

13) T. Nakabayashi and S. Kamiya, Nosan Gijutsu KenShi, 6, 149 (1959).

14) Y. Misawa, M. Matsubara and N. Doi, Nippon Shokuhin Kogyo Gakkaishı, 18, 326 (1971).

15) K. Koizumı and Y. Kidera, Yakugaku Zasshi, 97, 705 (1977).

16) P. V. Demarco and A. L. Thakkar, Chem. Communtcations, 2 (1970).

17) C. E. Johnson and F. A. Bovey, J. Chem. Phys., 29, 1012 (1958).

18) E. A Lewis and L. D. Hansen, J. Chem. Soc., Perkin Trans. II, 2081 (1973). 